\title{
Is Victory over Pancreatic Cancer Possible, with the Help of Tuned Non-Invasive Physiotherapy? A Case Study Says Yes
}

\author{
Pierre Le Chapellier, Badri Matta \\ Algology Department, Soissons General Hospital, Soissons, France \\ Email: pierre.lechapellier@ch-soissons.fr \\ Received 20 March 2014; revised 14 April 2014; accepted 21 April 2014 \\ Copyright (c) 2014 by authors and Scientific Research Publishing Inc. \\ This work is licensed under the Creative Commons Attribution International License (CC BY). \\ http://creativecommons.org/licenses/by/4.0/ \\ CC) (i) Open Access
}

\begin{abstract}
Could the conventional treatment of pancreatic cancer effectively be supplemented by a low level and non-invasive bio-electromagnetic treatment? A case study, based on the regular exposure of a patient to an electromagnetic field, EMF, emitted by a Rife-Bare technology device, suggests so. The plasma confined in a tube of this apparatus emitted radiofrequency solitons. These low level emissions were modulated by an "audio" frequency generator, pre-programmed for the treatment of this disease. After less than two months of exposure to these EMFs, the tumor completely disappeared in approximately two weeks. The explanation of the action mechanism includes a physics aspect relating to the properties of the dissipative soliton which is emitted-absorbed by any non-linear system, a biophysics aspect relating to the coherent structuring of the cellular bath by incident solitons, and finally a biological aspect. The latter is characterized by a critical resonance frequency leading the "unicellular" tumoral cell to adopt a self-destructive behavior. On the other hand EMFs with low level solitons have no effect on the tissues of complex multicellular organisms.
\end{abstract}

\section{Keywords}

Bioelectromagnetic, Cancer of Pancreas Therapy, Electromagnetic Fields, Immune System, Living nature, Rife-Bare, Soliton, Radiofrequencies

\section{Introduction}

In hospitals authorized for the treatment of pancreatic cancer, a non-invasive complementary treatment could be applied in order to improve the quality of life and to relieve the future pain of patients. Within this legal frame- 
work, a very welcome cure can come about, even if one swallow does not make a summer: the occurrence of an individual cure of a pancreatic adenocarcinoma, obtained with the participation of a complementary non-invasive bio-electromagnetic treatment, certainly does not mean that this complementary treatment has an objective therapeutic effectiveness. But, at a time when the optimal treatment of pancreatic cancer raises many questions, as recalled by Oettle, (2013), [1], anything that can enable such a cure invites reflection. The average lifespan of the patients is 6 months after they were diagnosed, as Wolfgang has indicated, (2013), [2]. According to Stathis, (2010), [3], this fact has two causes. On the one hand the phenotype of these tumoral cells is largely resistant to chemotherapy and radiotherapy, while having a strong potential for remote invasion. And on the other hand, and in addition, through lack of initial symptoms, $80 \%$ of the patients consult only when the disease has already largely progressed in them.

The disease starts with at least one cell becoming anaplasic, i.e. the cell behaves like a primitive unicellular being, out of control. According to Costa, (2012), [4], this is due, at the time of interaction between this cell and certain viruses, to the modification of proto-oncogenes. Instead of normally serving the essential cellular functions, they become oncogenes because they free the cell from its normal regulation. And so that such a mutated cell lives and multiplies among the normal cells, it is necessary also for it to adopt a mechanical discretion. According to Pradeu, (2007), [5], the antigens which a mutated cell presents to the immune system of the organism must not alert this system and lead it to a reaction of elimination. In a place selected to allow it to grow, and stripped of the symmetrical elegance which is peculiar to clusters of unicellular cells, (Cartlidge, (2013), [6]), a tumor will be formed slowly, sometimes over years, without symptoms. According to the theory of Pradeu, a tumor cannot grow quickly because if its growth were too fast, this rapid variation would induce a discontinuity in the presentation of its antigens to the immune system. And that discontinuity would cause the immune system to react mechanically to tumor elimination.

When the tumor is already well under way, the first symptoms appear, such as a persistent abdominal pain, an abnormal loss of weight, a problem with the sugar metabolism, a difficulty in digesting fatty substances. Then, when the tumor grows still bigger, it tends to obstruct bile circulation, which can enlarge the gall bladder and involve a yellowing of the skin and eyes, as Hammel, (2012), [7], has indicated. Once the blood vascular network of the tumor is well constructed, some cells do leave the pancreatic tumor and migrate in order to found tumors in other organs. Generally, this phase of progression, known as metastasis, is the cause of the patient's death in more than 90\% of the cases, (Ball, (2013), [8]). There is thus little chance to cure a patient whose disease has reached the metastasis stage and even less chance if the primary tumor has reached the mesenteric artery.

There are however new therapeutic opportunities. According to Oettle, they arise from the recent realization of the importance of the microenvironment in tumor propagation. A particular field of reaction which can reach $90 \%$ of total volume is located around the tumor. The compact fibrous tissue of this field interfaces the tumoral cells, the stromal fibroblasts, the pancreatic stellate cells, the endothelial cells, etc. An understanding of the ecological environment which interfaces these cells belonging to the whole of the tumor must logically make it possible to improve therapeutic action.

Also to be considered is the viral being's ecological microenvironment. This viral being, such as for example that of the hepatitis B virus, can be a cause of pancreatic cancer, Tabor, (2002), [9], Hassan, (2008), [10]. Its living microenvironment includes on the one hand the physical, chemical and biological presence of all the molecules which may have a role, and on the other hand the topological structures of the aqueous physical vacuum which appears to be around and between these molecules. Now it seems that some electromagnetic fields, EMFs, could modulate these intermolecular vacuum structures. In that way, specific EMFs could modulate the proteins' potentialities, for example the adenoviral protein $\mathrm{E} 155 \mathrm{kD}$, observed by $\mathrm{Xu},(2012)$, [11], or contribute to some structural resonance, and thus act on the active parasitic viruses, and thus involve salutary bio-effects.

According to Abbruzzese, (2007), [12], alternate or complementary methods of cancer treatment include bioelectromagnetic techniques. Indeed, as Payne indicates, (2013), [13], a hundred in vitro and in vivo studies on the anti-tumoral effects of low intensity EMFs were carried out in 2013. These low level EMFs can be applied in a complementary way, i.e. in combination with the other modes of oncological treatment, such as chemotherapy, X-rays, etc., or as an alternative. They have a defect: Their curative bio-effects are not systematically reproducible, because they depend particularly on the nature and the state of the cells. The most well-studied action mechanisms were on ionic balances, free radical production, interference with vascularization, and mitosis interruption. But physical action of EMF exposure on the structuring of the aqueous environment has also been stu- 
died, (Pomonarev, (2000), [14], Karimov, (2000), [15], Fesenko, (2002), [16]). As proteins only function when hydrated, (Guo Chen, (2009), [17]), their activation can thus be indirectly modulated by EMF exposure, which tends to involve a variation of signal transduction and cell signalization. This biological constraint could thus affect the living nature ${ }^{1 *}$ of micro-organisms. By admitting that their living nature really exists, the EMF bio-effect which causes a micro-organism seizure that can lead to a lethal resonance would have to be described more than in a merely mechanical way. Now this bio-effect, resulting from a specific EMF treatment, could allow elimination of the parasitic pathogenic viruses, the ones which are acting within the "mutated" cells, or those which are present in the aqueous external medium.

As stated by Jimenez, (2013), [19], there is clinical evidence that very low levels of radiofrequency EMF, amplitude modulated with "audio" frequencies, can cause therapeutic responses in patients with cancerous states. Jimenez showed that low level EMF, 27.12 MHz, modulated between $400 \mathrm{~Hz}$ and $21 \mathrm{Khz}$, causes significant tumor shrinkage. A summary of the observable mechanism is that exposure to these low level EMFs elicits an increase in the rate of intracellular calcium in the tumoral cells, so that tumor shrinkage would be associated with the IP3/DAG signaling pathway. The signaling variation in tumoral cells correlates with a tendency to autophagy and apoptose behavior. The IP3/DAG signaling pathway is the PI3K pathway which is generally involved in HCC, (Hepato-Cellular-Carcinoma) genesis. It would be this pathway which is typically modulated by these specific low level EMFs. The discovery that some EMF frequencies can modulate the living nature of pathogenic germs and tumoral cells in a lethal direction is due to historical work prior to that of Jimenez, in particular that of Rife, Crane and Bare, as presented by Lynes, (1993), [20].

This historical work led to recent studies of specific EMF bio-effects upon tumoral cells. They were carried out in vitro by Holland, (2010), [21], Bellossi and Dubost, (2008, 2010, 2012), [22]-[24], Dubost, (2013), [25]. Results showed that treatment of pancreatic cancer with non-invasive, low-level EMF is historically founded.

\section{Historical Empirical Work Resulted in Carrying out the Case Study}

During the second half of the 20th century, information on the inventions and work of the inventor Royal Raymond Rife, (1888-1971), might have seemed almost fictional, because of a wave of screening which had fallen on them. But towards the turning of the millennium several researchers found fragments of reports and machines. They started Rife's work again with current technology, and were able partially to confirm his results. It appeared thus more and more clearly that Rife worked methodically and in a reproducible way. Consequently his biological and curative results should be reproducible by means of devices and protocols resulting from his discoveries. The following historical description on this subject is based on texts and work of Lynes, [18], Scoon, [26], and Bare, [27].

From 1920 to 1929, Rife developed a "Universal Microscope". This apparatus used a cross play of two beams of ultraviolet polarized light. This made it possible to see, section by section, the live structures of micro-organisms, neither killed nor dyed, with an enlargement higher than $\times 20,000$. The visible color that the observed live structure took was a function of the difference between the two incidental invisible lights. This difference was to be regulated for each micro-organism to be observed. At the same time, the aqueous germs and their medium could be subjected to variable conditions of electromagnetic environment, in order to observe the EMF bio-effects on them. It thus appeared to Rife that the pulsed EMFs could have considerable bio-effects according to their frequency. Because each micro-organism would respond to a precise frequency, up to being destroyed, once entering a seizure, with agitation and change in form. When the specimen was exposed to an EMF flash at some Hertz of its resonance frequency, it reached a moment of seizure, and then recovered in a few seconds. But when the germ was exposed exactly on its critical frequency, it literally exploded. These effects, which were repeated thousands of times by Rife on germs and unicellular beings, never occurred in any multicellular tissues of complex organisms.

Rife's insight was thus to have established a specific mode of bio-electromagnetic communication between human thought and the living nature of simple bacteria. The main application was to selectively destroy pathogenic germs by exposure to specific EMFs, without damaging the healthy cells of complex organisms. Rife compiled a lengthy list of his test results of lethal frequencies for pathogenic germs. Then he tried hard to pro-

\footnotetext{
${ }_{1}^{1}$ Living nature is what is closely associated with living body biomolecules. It is said to ensure the organism's finality and its reactive behaviour to the medium conditions and events. According to Jung, [18], the living nature is the corporeal unconscious which makes any life proceed in a world made of images. The immunological reaction of a living organism would then not be merely a mechanical reaction to antigens.

*Bioelectromagnetic cure of a pancreatic cancer.
} 
duce a device emitting the appropriate EMF adjustably. He eventually invented an apparatus where the transmitter is an X-ray tube, modified by containing helium gas at low pressure, and not a vacuum. This tube was supplied by an amplified transmitter of radio frequencies which made the ionized helium electrically conducting and become an EMF transmitter. The lethal frequency was obtained by the product of a carried frequency modulation by a regeneration frequency. The lethal frequency thus comprised two parameters, the EM carrier frequency, measured in Hertz, and the regeneration frequency, measured by its wavelength in meters.

With the help of his first prototypes, Rife was able to cure animals having various specific infections, and study the different bio-effects. It appeared to him that tumor cells did not explode like bacteria, though they also died, but in another way. Rife then turned to viruses, (the infectious agents "which cross the filters"). After thousands of experiments, he formed the conviction that a cancer virus could be isolated and that his machine, by destroying the virus infects the tumoral cell, could cure cancer.

Then Rife succeeded in identifying two viral agents which should be, according to him, a source of cancers. He gave the name BX to the one causing carcinomas, (cancers of the membranes and skins), and BY the one causing sarcomas, (cancers of the bone, connective tissues or muscles). And, at the main point of all its research, he defined very specific EMF frequencies ensuring their destruction.

After showing his ideas and results to various doctors, in 1934 the latter organized a clinical trial at the University of South California, San Diego. Sixteen cancerous patients in the final stage were volunteered and were enlisted. The trial protocol planned an exposure, in the Rife private clinic, for three minutes every three days. But this amount had to be reduced because of strong reactions of the patient's organisms. The final result was that all were declared cured, 14 in two months, the last 2 in one month more, with a slight modification in the lethal frequencies, to adapt them to "mutant viruses". But the test was not a double blind test eliminating any influence of personal charisma. In addition, the dominant medical ideas at this time rejected the idea that EMFs could be active within the organisms, (Marino and Becker, (1986), [28]). There were differences of opinion within the committee, and they therefore refused to officially publish the dazzling results obtained, using as an excuse a need for complementary trials, which never took place. Then in 1944, the author of a report validating Rife's microscope was the subject of an attempted murder and he subsequently refused to mention Rife. Then a Rife associate, named Crane, invented a new variant of the machine, with a contact pad applicator. He proposed to use low frequencies in the audio range, between $120 \mathrm{~Hz}$ for tetanus and $2128 \mathrm{~Hz}$ for carcinomas. And very curiously these frequencies suggested by Crane in 1957-1963, based on those of Rife, seem to be usable for therapy with the machines with plasma tube which are today produced on the basis of $27.12 \mathrm{MHz}$ transmitters.

After the disappearance of Rife and all his work in 1971, an important revival was due to James Bare. He sought to simplify the Rife technology, too difficult to reproduce today. To supply the plasma tube, through some intermediate elements quite easy to find and regulate, Bare used 27.12 MHz CB transmitters, which can be directly modulated in the audio range. Bare thus managed to check Rife's results, including the explosion of germs under the bio-effect of a lethal resonance. To some extent he used the Crane low frequencies, derived from the original frequencies of Rife.

This Rife-Bare technology, which was quite easily accessible by means of Internet, such that, in 2013, there were ten thousand users of a Rife-Bare device, either self-manufactured from a kit, or marketed. And all kinds of accounts about individual treatments are available on an Internet chat room, [29] [30], though in the absence of any objective clinical trial. The majority conviction of the users is that this treatment actually works. The consensus on the therapeutic mechanism is that the low modulation frequencies used, lists of which for almost all diseases can readily be found, once transformed in the confined plasma, may have a lethal resonance in simple micro-organisms, (germs, viruses, bacteria), while being inoffensive for complex organisms, (with exception for a possible Jarisch-Herxheimer reaction).

\section{The Case Study of a Pancreatic Cancer Treatment Complemented by BioEM}

During February 2013, the patient E. S. noticed a slight epigastric pain when he was lying on the back, in bed, in the morning. The feeling was always in the same place, towards the duodenum. After speaking about it with his doctor, an abdominal ultrasound scan was prescribed, as well as a CT scanner. These two examinations were carried out on March 19. They showed the existence of a lump, of a size between 23 and $27 \mathrm{~mm}$, on the lower part of the head of the pancreas, towards the hook. This lump was attracting the mesenteric artery. On April 16, a biopsy was therefore carried out. It revealed that this larger growing mass was an adenocarcinoma, composed 
of tumoral cells. Then on April 19, a surgical operation discovered a bulky tumor of about $4 \mathrm{~cm}$, which was impossible to remove because of its too close relation with the superior mesenteric artery (see Figure 1).

The only conventional therapy remained chemotherapy which was quickly started, with four sessions of Folfirinox administration, spaced two weeks. The patient's good tolerance made it possible to prolong the treatment by three sessions, during summer. In September 23, a scanner examination revealed that the infiltrated tumor near the pancreas hook was reduced to a size of $12 \mathrm{~mm}$, which testified a clear, but limited, effectiveness of chemotherapy.

Previously, August 25, 2013, the patient E. S. had received information about the Rife-Bare systems. He thus discovered the historical possibility that a non-invasive bio-electromagnetic cancer treatment exists. In the first days of September 2013, the patient ES decided to e-mail the Canadian company which produces the PERL apparatus using a marketed Rife-Bare technology, [28].

After the first exchanges, a choice appeared: He could either import an apparatus, or purchase a new one from a French private individual whose relative was deceased before the apparatus could reach him. This fact raises an important point: The choice of this kind of complementary treatment is best carried out as soon as the pathology is diagnosed and thus before chemotherapy starts. For the program of the sets frequencies to be used with the device includes an emission protocol intended to boost general health. The session that begins the treatment must use this, because it can help users who may encounter noticeable detox symptoms. On the other hand, around two months of daily exposure are needed before an anti-tumoral bio-effect could appear.

In the first days of October 2013, the patient ES purchased the new apparatus from the private individual deceased before reception, while taking a contract with the firm for training as user and for a personalized follow-up by "hot line", on Internet.

He began his bioelectromagnetic treatment on October 04. Soon after the first week, he noticed a net gain in wellbeing, improvement of sleep and a little lessening of his diabetic problems. Then, in agreement with his surgeon, he chose to test his machine one more month before a clinical re-examination for a possible resection. At the end of six weeks after the beginning of this EMF physiotherapy, the tumor size had not changed but the tumor's relation with the mesenteric artery had evolved. On November 7, an ultrasound scan-endoscopy under general anesthetic showed the stabilized $12 \mathrm{~mm}$ tumor, but with a small edging interfacing it to the artery. From then on, things improved quickly.

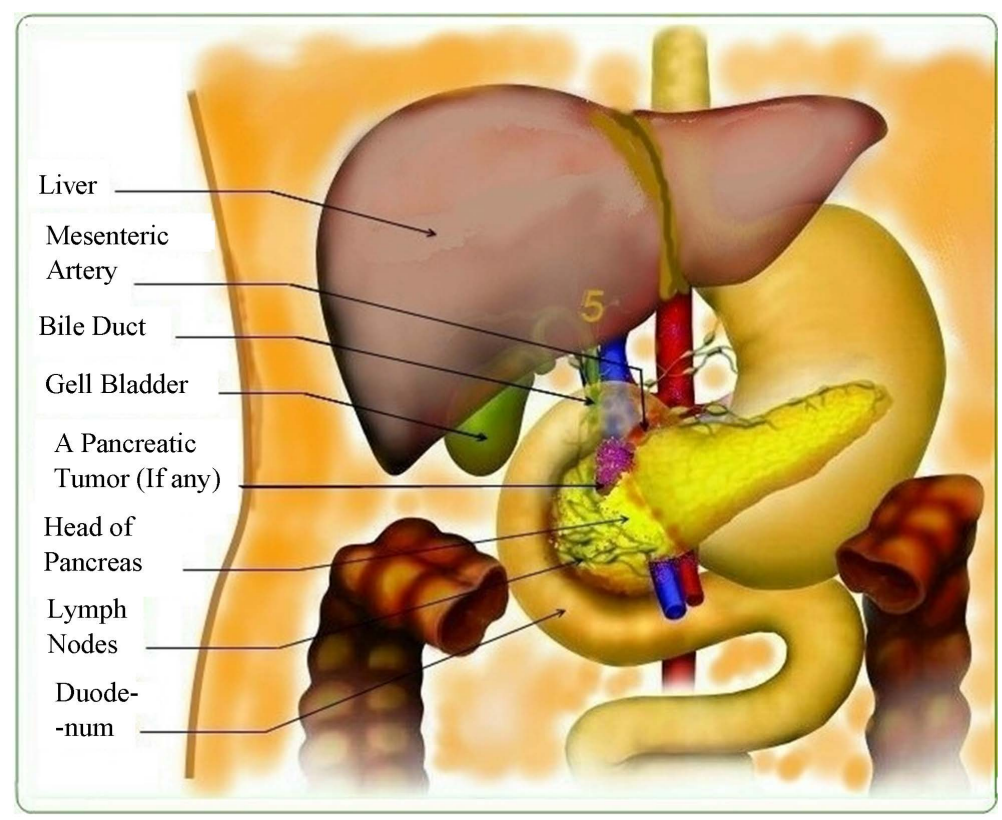

Figure 1. Location of the pancreatic tumor. The head of the pancreas is the large rounded part of the pancreas, on the right side of abdomen, on waist level (The patient's tumor was in fact situated more in the lower part of the pancreas head). 
On November 21, a CT-Scan revealed that the pancreatic tumor had become indistinguishable, but that a secondary tumor, size more than $2 \mathrm{~cm}$, could have appeared in liver segment 5 . To discover more about this, a PET-Scan was carried out on December 6 and an MRI on December 9. The PET-Scan revealed there was no hypermetabolic area on the liver, but a biliary cyst in continuity with the cystic channel. The PET-Scan also established that there was no longer any hypermetabolic area on the pancreas, apart from an area corresponding to a nonspecific fixing to the duodenum. The IRM then confirmed the absence of any detectable ${ }^{2}$ anomaly on the pancreas. The overall evolution of the tumor is represented in Figure 2 outline. Figure 3 shows before and after imagery comparison.

On beginning of December the patient had no more troubles and he had returned to a full quality of life, though he was still concerned about a tumoral marker. For although in January 2014, his level of CEA marker, (carcinoembryonic antigen), had fully returned to normal, that of CA 19-9, (cancer antigen 19-9), rose a little, by 30 to 800. And even though the level of CA 19-9 does not make a very high contribution to the diagnosis, (HAS, (2010), [31], and Lévy, (2007), [32]), it indicates that there is still a certain form of activity. The cure of patient ES will really only be final when this level of CA 19-9 has returned to normal.

The orientation of the patient's living nature towards this final cure, rather than towards a relapse, is attested by a CT-Scan of February 04, 2014. The images shown in Figure 4 indicate that secondary aspects of pathology, such as tiny infiltrations of grease located between the pancreas head and hook, and tiny infiltrations of liver tissue, are all in clear regression, without the use of any medication.

Thus the conclusion of this unique case study is that a non-invasive bio-electromagnetic treatment, complementary to chemotherapy, applied for two months by means of a PERL device equipped with a confined plasma tube, (a tube emitting EMF frequencies according to Rife-Bare technology), and whose carefully chosen emis-

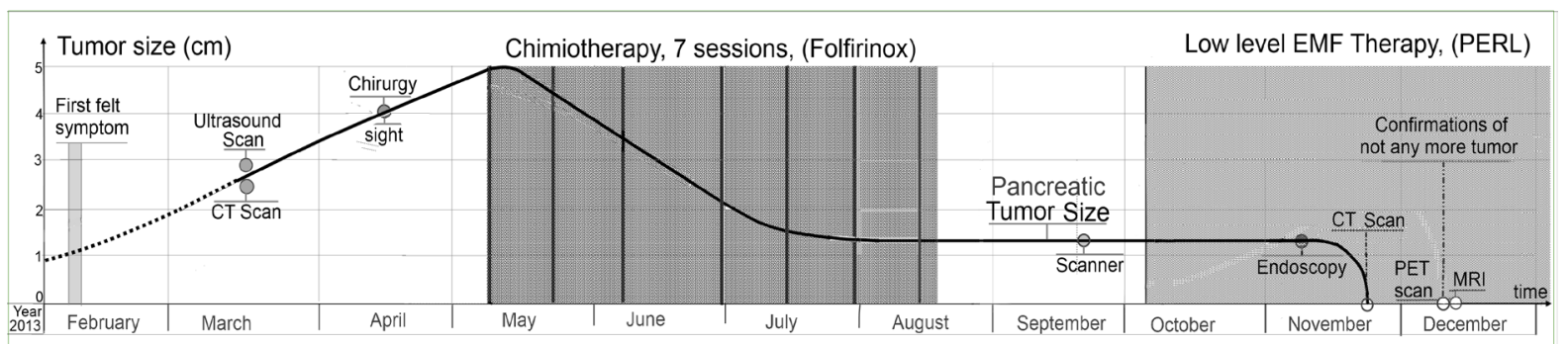

Figure 2. Schematic evolution of the patient's pancreatic tumor towards healing, (a case study) The outline of this cancer evolution shows that the complementary EMF treatment with the PERL device, applied after 7 sessions of chemotherapy which resulted in a stabilized reduction of the tumor, required to be used for less than two months before disappearance of the tumor in less than 2 weeks.
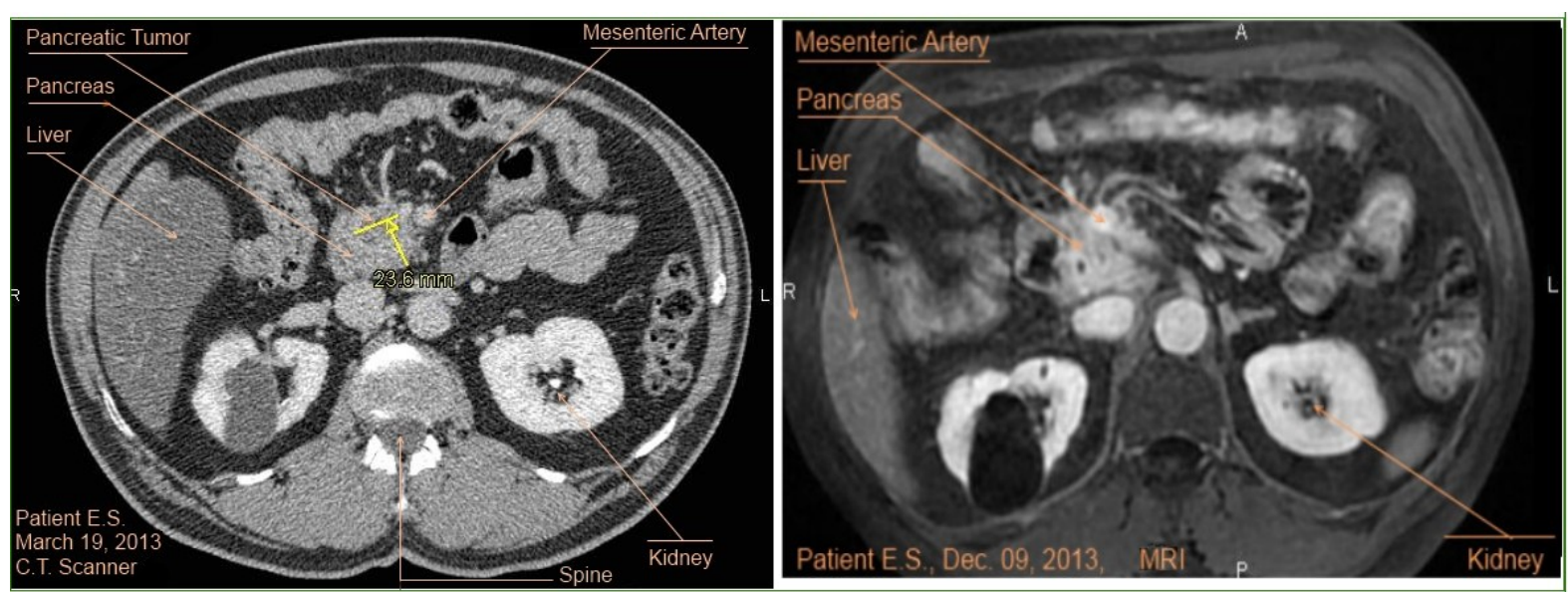

Figure 3. Comparison between CT Scanner Image, March 19, 2013, (left) showing the pancreatic tumor, and MRI Image, Dec 09, 2013, (right) showing not any tumor. Imagery showed that the $23 \mathrm{~mm}$ pancreatic tumor which was adjacent to the mesenteric artery on March 19, had entirely disappeared by Dec. 09, after the EMF treatment with PERL device. 


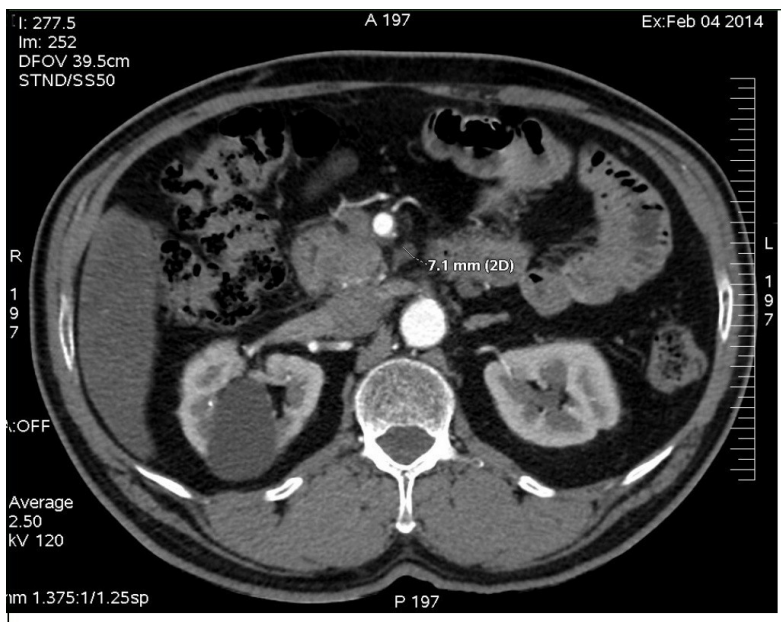

Figure 4 CT Scanner Images, Feb 04, 2014 showing no tumor and a regular decline of secondary infiltrations in grease and liver

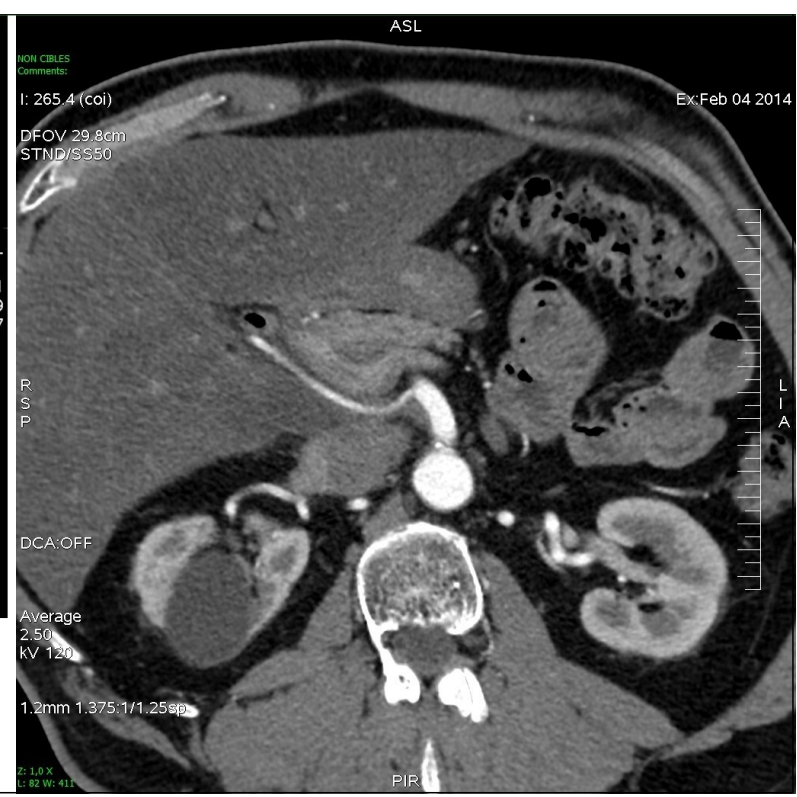

Figure 4. CT-Scanner images, Feb 04, 2014. Images showed that, two months after the disappearance of metastasis, the secondary aspects of pathology, such as tiny infiltrations of the grease located between the head and the hook of the pancreas and tiny infiltrations of the liver tissue, are all in clear regression, without intervention of any medication (The small ganglion of $7.1 \mathrm{~mm}$, apparent on the image on the left, is not tumoral).

sions were tuned by a personalized follow-up, caused a disappearance of the patient's tumor associated with a regression of secondary infiltrations, constituting an undeniable victory over the pancreatic cancer.

The description of the action mechanism of this salutary treatment requires three successive approaches, the first according to physics, the second according to biophysics and finally the third according to biology.

\section{Approach of the Treatment with the Perl/Rife-Bare Device, According to Physics}

\subsection{The Device}

The PERL device is simpler to use and less cumbersome than previous prototypes according to the Bare or worse, the Prioré ${ }^{2}$ technique. The Rife-Bare prototypes, constructed around the year 2000 for the laboratories, comprised an adjustable function generator, a $27.12 \mathrm{MHz}$ transmitter, an amplifier, a tuner for antenna with a balun and finally an antenna made up of a tube with confined plasma, [24]. The PERL device, marketed by Resonant Light Inc., incorporates the components of the Rife-Bare device: The basic case measures $45 \times 33 \times 13$ $\mathrm{cm}$. It includes the transmitter $27.125 \mathrm{MHz}$, the power amplifier and the tuner.

As Figure 5 shows, this case is surmounted by a lead glass tube, filled up with argon under low pressure, $(0.026 \mathrm{~atm})$. The emission power of this tube, when the plasma, (the ionized gas), is conductive, is 60 Watts.

The case is connected by a cable to a frequency generator which is programmable using a keyboard. This generator also contains a set of preprogrammed banks of frequencies corresponding to the treatment of specific pathologies, according to protocols worked out by Tunney, (2012), [36]. It produces waves which can be swept around an average. The waveforms of the waves are adjustable by the user. However, rectangular waveforms with 70/30 duty cycle are preferred, because they best support the emission of harmonics by the confined plasma, [27].

Contraindications: As a basic effect of the exposure seems to be to reinforce the reactions of the immune system, the use is contraindicated for patients having received transplants or stem cells. For EMF questions, use of the device is not recommended for patients with defibrillator or pacemaker before 1992. It is it also, for safety reasons, not recommended for pregnant women and young children.

${ }^{2}$ In 1969 the Prioré process for treating cancer used a combination of Pulsed Magnetic Fields and EMF transmitted from a large bulb with plasma. Patented in 1963 in France and 1966 in the USA, and partially evaluated in France, it has not been used in any current clinical practice, [33]-[35]. 


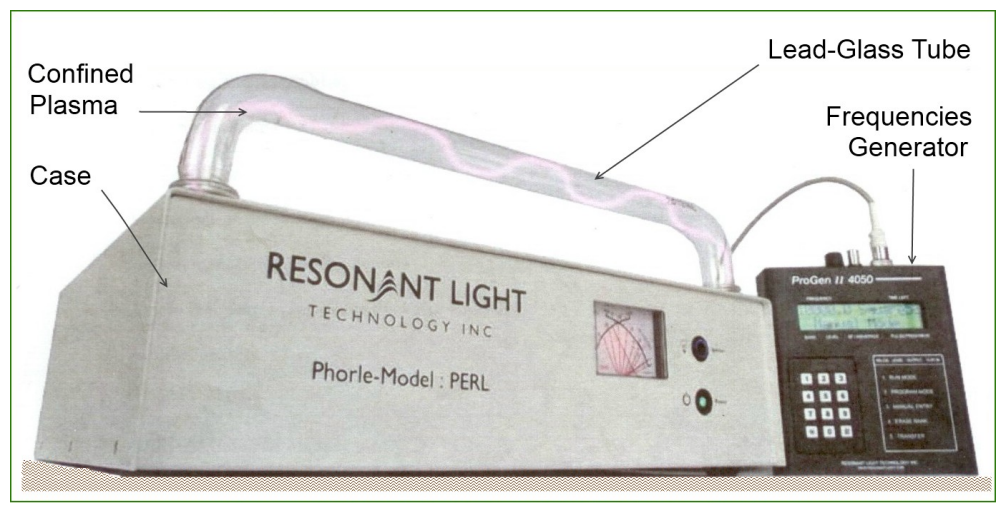

Figure 5. The PERL device, with its frequencies generator.

\subsection{The Guiding Principles}

The first guiding principle: is described by plasma physics. Under the influence of a strong electric field, a partial ionization of the atoms of the inert noble gas confined in the tube produces a conducting medium called plasma. This is composed of positively charged particles (ions) and negative charges (electrons). As soon as an electron escapes from an atomic layer, this plasma will begin to oscillate, according to a collective movement where each plasma particle adopts a position such that the total force of electric interactions constantly remains null. This phenomenon involves EMF emissions whose sources are dipoles of ions with different charges.

Thus the guiding principle of the treatment with a Rife-Bare apparatus resides in a confined plasma emitting solitons originating from the ionic discharges. Mathematically, a soliton is the solution of a non-linear differential equation. It is also a discontinuity described by the thermodynamic topology which is based on the external differential forms.

According to hydrodynamic physics, a solitary wave which propagates by preserving its form and its speed, a soliton, can be described by the nonlinear differential equation of Korteweg de Vries, (1895). It is admitted this equation has solutions with solitons ${ }^{3}$ because the two opposing phenomena in its physical background, creation of collisions and dispersion, are compensated by producing a balanced state.

According to plasma physics, it is from the electric potential appearing between two close ions that non-linear effects of collision and dispersion are created and compensated. They give rise to solitons at the moment of the electrical disruption. The dispersion relation links the pulsation $\omega=2 \pi \omega \mathrm{F}$, and the wave vector $\mathbf{k}$. The emissions depend on the electric field $\mathbf{E}$, the magnetic field Band the disturbance of the ionic density. If $\Delta \mathrm{n}$ is the minimal ionic density required to produce a soliton, if $(\Delta \mathrm{n})_{\mathrm{f}}$ is the necessary ionic density to produce the whole of $\mathrm{N}_{\mathrm{S}}$ solitons, and if $\mathrm{f}$ is the frequency applied to the current $\mathrm{J}$ which circulates in plasma, there is the equality: $(\Delta \mathrm{n})_{\mathrm{f}}=$ $\Delta \mathrm{n} . \mathrm{N}_{\mathrm{S}} \approx \eta / \mathrm{f}^{2}$. As this density $(\Delta \mathrm{n})_{\mathrm{f}}$ is also related to the frequency $\mathrm{F}$ of plasma collective oscillation by: $(\Delta \mathrm{n})_{\mathrm{f}} \approx$ $\kappa . \mathrm{F}^{2}$, we have that the frequency $\mathrm{F}$ of the plasma oscillations is inversely proportional to the applied modulation frequency f, with: $\mathrm{F}=\eta / \mathrm{\kappa f} \approx 2.35 .10^{12} / \mathrm{f}$, for a given type of tube. For this model, Dubost, [24], predicted that: $\mathrm{F}$ $=11.7 \mathrm{GHz}$ for $\mathrm{f}=200 \mathrm{~Hz}$, and that $\mathrm{F}=240 \mathrm{MHz}$ for $\mathrm{f}=10 \mathrm{kHz}$.

The modulation frequency $f$ defined in the function generator is thus transformed into an emission of frequency F solitons, characterized by a transverse electric field Es. Through induction measurement, Dubost indirectly measured Es at a distance of $0.4 \mathrm{~m}$ from the tube. He found a value of about $1.6 .10^{10} \mathrm{~V} / \mathrm{m}$ at frequency $\mathrm{F}=$ $1 \mathrm{kHz}$ and $1.4 .10^{8} \mathrm{~V} / \mathrm{m}$ at $\mathrm{F}=10 \mathrm{kHz}$. In spite of its decline according to the cube of the distance, such an elec-

\footnotetext{
${ }^{3}$ Soliton: In their work on nerve impulse propagation, Hodgkin and Huxley observed that an excitation threshold is necessary to produce a visible phenomenon, and that, above this excitation threshold, the signal which appears has a constant form and is propagated at constant speed. Hodgkin and Huxley conjectured that this behavior was due to diffusion and nonlinear interaction between an electrostatic potential and reactions of several ions between them. Thus this mechanism was controlled by a system of reaction-diffusion equations (Similar equations can apply to the plasma emissions of the PERL tube). Mathematical work then consisted in showing that solutions of the HodgkinHuxley system had a behavior in conformity with experiments, Bardos, (2012), [37].

A soliton is a solitary wave, a discontinuity, which is generally based on complex wavelets. As a package of waves or a pulsation, it maintains its form while moving at quite constant speed along a path. A typical example is the solitary wave which reaches the mouth of a river. It exists by cancelling the usual dispersive and nonlinear effects in the medium. Mathematically, solitons are solutions of partial differential equations which are non-linear and slightly dispersive. Optical solitons, or Photons, can combine various frequencies and make it possible to transmit a lot of information. They ensure endurance of bio-systems, (observed on the level of the collective low-frequency movements in the DNA and the deployment states of proteins).
} 
tric field remains important because it is sourced by a disruption between two ions. If the soliton has very little power because it is a point, its electric field can nevertheless cause a longitudinal resonance in the microtubules of tumoral cells exposed in vitro. Nevertheless it is not certain that this electric field Es is the main biologically active agent for exposures in vivo, with the PERL device. It appears to be the active agent with the device of Kirson, (2009), [38]. But the Kirson TT waves have an action principle which is different for a use which is similar, that of a complementary treatment of cancer by means of radiofrequencies.

According to the topological thermodynamic approach, the radiofrequency solitons propagated in space out of the tube are discontinuous signals. They can be named as Photons, because they are electromagnetic waves of frequency F meeting Maxwell's wave equation. But they are also solitary topological defects propagating in the physical vacuum, therefore they are spinors, (complex direction fields,) which satisfy the null eikonal Equation (4), [39].

These solitons are thus discontinuous radio frequencies signals which are more than merely waves. Such active agents differ from the visible or infra-red light which is emitted by the tube. Because that light is only a wave which is a solution of the wave equation, it is not a signal with curative capacity. According to Kiehn, (2013), [40], the structuring of intermolecular space (which is the background of the extracellular aqueous medium) depends on the self-similarity density $\mu$ of the "defects" which are in this physical vacuum. This density $\mu$ may be gradually built up, because the energy field domain of the physical vacuum is complemented by the evolutionary kingdom of the coherent "defects" of its structure (See Figure 6).

The concept of topological coherence thus differs from the concept of coherent light, since it is referred to the existence of recognizable properties which are maintained through deformations, together with a long evolutionary lifespan associated with solitons. Thus in addition, this idea of topological coherence easily fits with the antigens presentation at the Immune System. Because, according to Pradeu’s theory, [6], when Immune System recognizes these antigens as a continuous property, even through progressive deformations, it does not react.

A specific frequency: According to the description of Akhmediev, (2004), [41], the stable structures in nonlinear non-equilibrium systems emit specific dissipative solitons. The solution of the equation which de-



Figure 6. Solitons signals as coherent defects in an electromagnetic wave field. Optical solitons are coherent defects which are, in the 4D physical vacuum, complementary to the energy wave field. They can be condensations of particles, or field discontinuities, or solitons. These spherical discontinuities which are propagated include an outer wave front "On", an internal wave front "Off”, a center $\Phi$ and a direction vector. Each forms a signal whose coherence increases according to the density of self-similarity.

\footnotetext{
${ }^{4}$ The eikonal equation: This equation is a PDE, a partial derivative equation, which goes beyond the Einstein-Minkovski space time invariant known as: $\mathrm{ds}^{2}=\mathrm{dx}^{2}+\mathrm{dy}^{2}+\mathrm{dz}^{2}-\mathrm{c}^{2} \mathrm{dt}^{2}$. It can be written: $\left\{ \pm(\partial \varphi / \partial \mathrm{x})^{2} \pm(\partial \varphi / \partial \mathrm{y})^{2} \pm(\partial \varphi / \partial \mathrm{z})^{2} \mp(\partial \varphi / c \partial t)^{2}\right\}=0$. The density $\mu$ of external differential forms being:

$\mu=r . W\left\{d x^{\wedge} d y^{\wedge} d z^{\wedge} d t\right)$, the wave equation of the soliton $F$ is: $r=\varphi\left(x^{k}\right)$, (Kiehn, 2012, [39]).
} 
scribes this emission results from a double equilibrium between, on the one hand non-linearity and dispersion, and on the other hand loss and gain of energy. By admitting that such structures could correspond to the microorganisms which are tolerated, or ignored, by the Immune System (because of a continuity in their antigen presentation), the theory of Akhmediev can explain why each type of living micro-organism in the organism has aspecific resonance frequency: depending on the actual bacterium structure, this frequency should be accorded to the frequency of the dissipative solit on emitted by a bacterium in an isolated state. This idea also leads to the question of the infrared photons which are emitted and absorbed by cells.

According to the optical approach of Popp, (2004), [42], when a culture bath without the cells emits an intensity of infrared photons, the living cells placed out of this bath emit another intensity of infrared photons. But the whole of the cells replaced in their bath emits much less photon intensity than the sum of the two separate intensities. This tends to prove that cells absorb, (suck in), external photons.

Here Popp specified that a coherence of the external photons is necessary for their absorption. If the coherence length of the external domain is less than the cell's size, sucking in could not play an active part in the cell biochemistry. It is thus necessary that the coherence length $L$ of the domain be equal or superior o the cell's size, so that energy sucked in could bring about a quantitative effect. This energy is thus used for excitation of the electrons and proton spins of the molecular matter, which modulates the activity of proteins, and hence the cell biochemistry and micro-organism itself.

To increase the coherence length L, photons, or photons-solitons, need to be actively stored in the domain, in agreement with the laws of quantum mechanics. This phenomenon is possible, at the time of phase transition from chaotic states towards coherent states, when the photons can be accumulated into a coherent state, but at lower energy levels than in the previous state, at lower harmonic frequencies.

A quantum approach of non-linear systems was developed by Mesquita, 2006, [43], from the soliton theory of Davydov. According to this theory, the density of coherent solitons considered as photons results from a Bose-Einstein condensation. This condensation may last if it includes a Fröhlich effect. This effect resides in the appearance of lower harmonics, therefore of lower energy levels. Such a Fröhlich effect thus corresponds to the "active storage" effect described by Popp. Over a certain time, there is thus a possible accumulation in the aqueous bath of the cells and micro-organisms of the "coherent defects" which are solitons.

Storage in bath can occur only at lower harmonic frequencies than the initial frequencies of solitons propagated from the confined plasma, during a certain time. An increase of the self-similarity density $\mu$ may then occur, so that, in Popp language, the coherence length $L$ of the external domain increases. When this length L exceeds the size of the micro-organism, the effect of coherent energy absorption, accorded to the law of Kirchoff ${ }^{5}$, can appear. A soliton frequency may then more easily become recognized, when it is tuned to the proper frequency of the micro-organism structure, i.e. with that of its dissipative soliton. This explains why accumulation of exposure to specific frequencies can lead the pathogenic germ to a seizure, right up to its destructive explosion. Although the lifespan of isolated solitons is extremely short and their energy strongly absorbed by aqueous mediums, a plasma tube emitting solitons with specific frequencies can thus constitute a physical source of nonthermal biological effects, able to act at some distance on germs and unicellular beings and not on multicellular beings' cells.

\section{Biophysical Approach to Cancer Treatment by Means of Solitons}

Space structuring induced by solitons signals which are emitted by a plasma tube appears to allow, according to the frequencies $\mathrm{F}$ applied in the aqueous medium, a selective destruction of various simple micro-organisms. Dubost studied and checked successively three biophysical assumptions to explain this destructive phenomenon.

\subsection{Collective Movements of DNA}

An initial approach of Dubost, [24], was that the solitons frequency F may structure the aqueous bath of the bacterium DNA. The resonance prediction was calculated according to the number of nucleotide pairs in DNA and this number is specific to each micro-organism. But such a calculation predicted theoretical frequencies whose values were up to $100 \%$ different from the measured effective lethal frequencies. Another explanation

\footnotetext{
${ }^{5}$ According to the Kirchoff law, for the same wavelength $\lambda$, the emissivity and the absorptivity of a given material are same and equal to the product of the traversed absorbing mass by the absorption coefficient.
} 
was thus necessary.

\subsection{Protons Spins}

Dubost then observed that the basic lethal frequency $\mathrm{F}$ can be of the same order of magnitude as the magnetic nuclear resonance frequency $v$ of the proton. This frequency $v$ is given by the equation $v=2 B_{0}$. $\mu / h$, where $B_{0}$ is the ambient magnetic field induction, in Teslas, $\mu$ is the ion magnetic moment, and H Planck's constant. For the $\mathrm{H}+$ proton, the frequency is: $v=4 \cdot 25 \cdot 10^{7} . \mathrm{B}_{0}$, in Hertz, that is $\mathrm{n}=2125 \mathrm{~Hz}$ for geomagnetic induction $\mathrm{B}_{0}=50$ $\mu \mathrm{T}$, (or 0.5 Gauss). This frequency value is indeed very close to the low frequency $2128 \mathrm{~Hz}$, given by Crane to support the destruction of the viruses causing sarcomas. Nevertheless the idea that a direct physical action on the proton structures may cause an explosion of the viruses was proved unsatisfactory. But the physical action of the soliton frequencies on the spins of the mobile protons $\mathrm{H}_{3} \mathrm{O}+$ of the aqueous medium structure remains possible. This assumption was checked at the time of destruction of various bacteria, [22]-[24].

\subsection{Microtubule Resonance}

The third explanatory approach of Dubost, (2013), [25], concerned a possible mechanical resonance of the microtubules which are parts of the centrosome skeleton of the tumoral cell. In the case of pancreatic tumoral cells, it was calculated to predict a lethal frequency which closely corresponded to the in-vitro tested frequency. This frequency was also close to the frequencies of Kirson's TT waves.

But subsequently, Dubost, (2014), [44], discovered specific antitumor frequencies. They act by a mechanical disturbance resulting from a depolarization of the astral microtubules, at the time of mitotic spindle formation, for specific tumoral cells. These frequencies are included among the modulation frequencies that Pasche, (2013), [45], indicated for the treatment of cancers. They are between $6.65 \mathrm{kHz}$ for the cell Hep 3B, and $19.76 \mathrm{kHz}$ for cell MCF7.

To be active these lethal frequencies require an electric field of about $10^{5} \mathrm{~V} / \mathrm{m}$. Yet at $0.4 \mathrm{~m}$ of the transmitting tube the electric field of each radiated soliton is locally $1.4 .10^{8} \mathrm{~V} / \mathrm{m}$ when their frequency is $10 \mathrm{kHz}$. The relationship between the two values is $63 \mathrm{~dB}$, which is enough to compensate weakening at the time of in vivo exposures. But this biophysical microtubule resonance does not explain why tumor destruction requires two months of exposure of the full patient's body to the PERL device emissions before appearing. An additional description to the microtubule resonance theory thus remains necessary.

Now, the microtubule resonance theory apparently offers a bridge to this psychical aspect of simple organisms which belongs to their living nature. For it all comes back to the psychophysical interaction model based on microtubules which was proposed by Penrose and Hameroff, (1995, 1998), [46] [47]. This model was partially readopted by Albrecht-Buehler, [48], precisely for the straight astral microtubules which connect the cell centrosomes to membrane, with the statement that centrosomes should be the psychic center of cellular intelligence. That being said, Albrecht-Buehler observed two things: On the one hand microplasts which detached from the cells and which travel in the external medium also make choices. And on the other hand the aggregative phenomenon of unicellular beings depends on the dispersion of the infra-red light they are emitting in their external aqueous medium.

\subsection{The Coherent Structuring of the Aqueous Medium}

By admitting that the universal primary physical target of EMF low level signals should not be physically within the cell, because it is neither centrosome, nor microtubules, nor ADN, nor protonmolecules, it may be the spins of the protons which produce moving defects in the hydrogen bond network of the external aqueous medium structure, [49]. According to Karimov, 2000, [15], biological systems are indeed aqueous and their biomolecules are hydrated. The state of hydration can depend on non-linear coherent structures which become significant in non-equilibrium systems. By its progressive action on the proton spins, the incidental signal of a soliton acts while being stored coherently in this aqueous target. This soliton which can become durable, [50], is topologically a photon. This photon is defined as a singularity which is sensitive to the space polarization and chirality states with which it can have an interaction, [51].

This concept can be related to the first of Rife's discoveries about the effect of crossed beams of polarized light upon living micro-organism structures. While deflecting these beams chirally, these substances emitted 
visible light from their absorption of crossed polarized ultraviolet light. The point which brings about the involvement of living nature, is their ability to enter a seizure, or even self-destruct, with perception of resonant radiofrequency signals. This role of the living nature of a virus or a unicellular species is suggested by Albrecht-Buehler in terms of an intelligence, with some naive mechanical ontology. This idea poses problem. Conversely, the philosophy of Nature affirms that the biological reaction of any unicellular being is non-mechanical, [53]. This concept agrees with the theory of the living nature proposed by Jung.

\section{The Mode of Biological Resonance to a Signal with Low Level Solitons}

\subsection{Living Nature}

According to Jung, [53], the living body is an arrangement of matter adapted to make a living being possible. The living body is thus a bio-matter system which is ready for life and not a simple cluster of matter. But it cannot live without the intervention of living nature, because any inanimate body lacks something essential for life which is psychical substance, or psychical energetics. Here, Jung crossed the barrier between psychical and physical, stating that any immediate life operates in an image world, [54]. It follows that the living nature of any organism in a situation produces some "images" of adaptation and reaction,-in relation to a natural psychical conflict existing on the most intimate level. This conflict is due to a (dialectical) opposition between pure instinct and inherited mass of possibilities, [55]. The "image" translates the sense of that instinct which takes on the form of a situation, [56]. But the pure instinct of any living organism, simple or complex, is an impersonal biological phenomenon which is collective, [57].

Cellular biology supposes that the constant remodeling ofsignalsomes ${ }^{6}$ and signaling networks is due to the variable conditions of the medium. But this remodeling could result as much from a perception according to instinctualsense order cascading the transduction of physicochemical messages according to causal order. The perception that a unicellular species can have does not form a representative image as it does with complex beings. What happens could be only an architectonic stress ${ }^{7}$ resulting from its extension in the external 4D topological structure, [58]. When this overall stress acts particularly in the structure subjacent to signaling networks, it can also act to modulate the mechanical structure of a germ and thus change its dissipative resonant frequency. Figure 7.

\subsection{Is the Response of a Germ to a Frequency Due to a Non-Mechanical Resonance?}

Because the oscillatory electric field is strongly absorbed by an aqueous medium, there can be some difference between in vitro and in vivo treatments by means of solitons. During in vitro exposure, polar or ionized biomolecules are agitated due to the electric field component, with a possible thermal effect. But in vivo, at a depth of $10 \mathrm{~cm}$, their mechanical agitation by the electric field component of the signal can be negligible. What remains on the other hand is the magnetic field component of the signal. The action of this component on the mobile protons which constitute active defects of the water structure has been described, (Pang, 2006, [59]; Binhi, 2007, [60]). In this way, the structuring effect of propagating solitons can be accumulated in the energy structure of the 4D aqueous physical vacuum, with an increasing coherence level which is described by thermodynamic topology in term of self-similarity density $\mu$.

The local bio-effect of a whole body exposure to radiofrequency solitons is thus primarily a qualitative bioeffect depending on the existence of $a$ relational energetics specific to the living nature of viral or unicellular beings. Because its living nature is closely linked to its living body, the phenomenon of germ self-destruction by apoptosis, autophagy or explosion, should be that of a supra-mechanical resonance.

The germ's relational energetics is observable empirically: According to Tunney, [60], the virus "is able to change its frequency" either at once when too close a frequency fills up its external energy medium, or through memory because of a treatment with too regular a schedule: The effectiveness of the applied resonant signal is greater if it happens in the form of an "inevitable" event in the 4D non-being of the pathogenic germ. Effectiveness is reduced when a signal is expected. This reactivity of the micro-organism is related to a non-mechan-

\footnotetext{
${ }^{6}$ The signalsome is on the one hand the system of signalling components resulting from the genome, specific to each cell, and on the other hand any dynamic protein complex on a signalling path.

${ }^{7}$ Architectonic: having an organized and unified structure.
} 


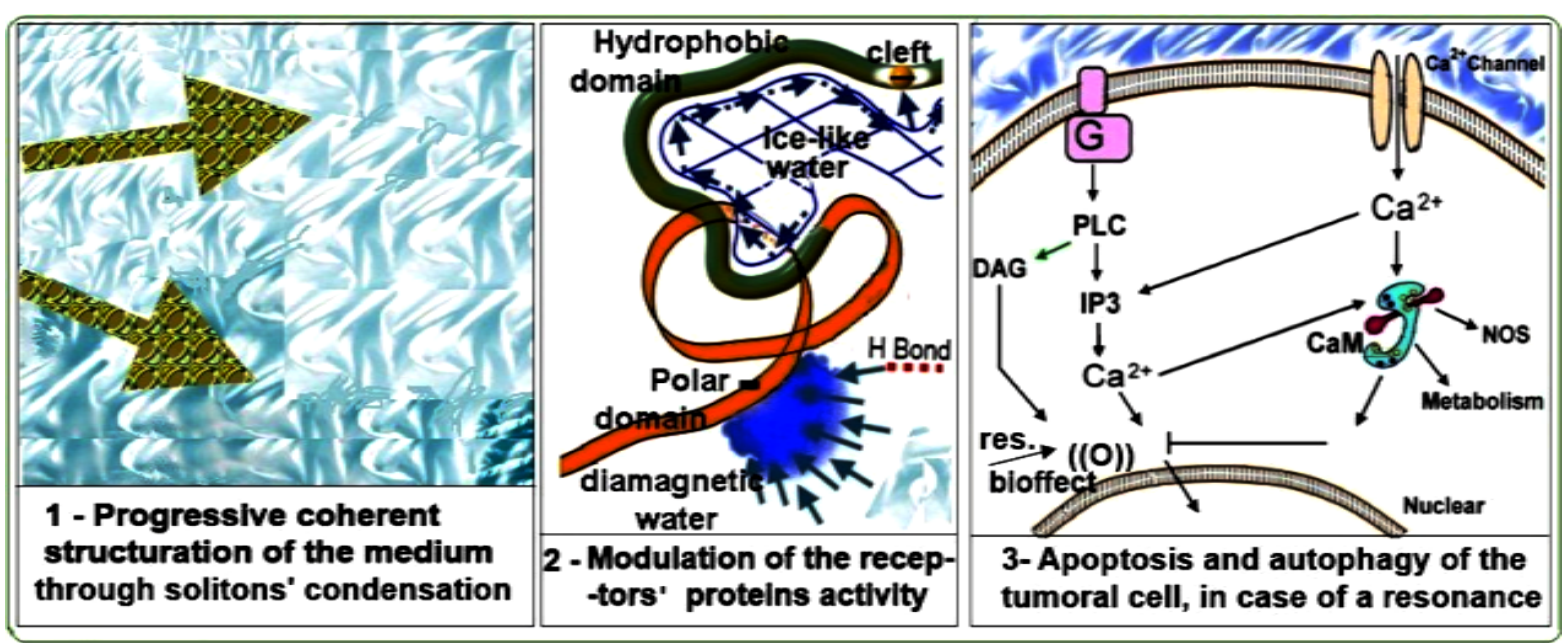

Figure 7. Proposed outline for the action mode of resonant solitons on tumoral cells in vivo. The suggested outline for the soliton's action on tumoral cells in vivo links the causal mechanical aspects to the energetic bio-effect resulting from living nature. First of all, soliton condensation may allow a coherent structuring of the external aqueous medium. Secondly, through protein hydration, this structuring modulates the activity of the cell receptors. Thirdly, it modulates the DAG/IP3 signaling path. It facilitates the lethal resonance bio-effect which in fact could depend on the living nature of the tumoral cell.

ical resonance due to relational energetics ${ }^{8}$. According to the philosophy of nature, relational energetics is linked with the functional non-being of the living beings, [61].

\subsection{Epistemological Definition of the Functional Non-Being}

The idea of the functional non-being differs radically from the non-self concept in the classical theory of the Immune System, because it is what allows the evolutionary movement of all that is oneself. The description of movement by Galileo in terms of kinematics left aside the significant relations that living beings have with their inhabited medium extended as a non-being. But the living organism, unicellular or complex, adopts different states and behaviors, and even evolves epigenetically, according to this perceived sensitive medium which is whatever it itself is not.

In this connection, the logical paradox of Parmenides claimed that in a finished and full universe, being is, and non-being is not. This paradox was broken since physics showed that everywhere between atoms an intermolecular vacuum exists which is an active non-being (Its biophysical energy field for example comprises a hydrophobic force field and a Van der Waals force field). The intermolecular vacuum is thus a real non-being which could constitute that ecological environment which enables evolution.

The philosophical non-being: Yet Aristotle held that the movement which allows a living being to evolve, i.e. to progress from one state to the next, is determined only by its non-being. Now, Jung held that the organism's psychic potential energy, which corresponds to the attitude it has that directs its psychic forces, is equal to the exact sum of its beingand non-being. In the same way, during the $20^{\text {th }}$ century, the philosophy of Nature according to Whitehead, (1920), [62], followed by Merleau-Ponty, (1959), [63], connected the concept of the evolution of process with that of non-being. Such non-being includes the 4D topological envelope, as well as the all of the being's potentialities. Epistemologically this non-being can be reduced to the structure of the being's 4D ecological environment, i.e. the structure of its extracellular aqueous physical vacuum. It endows the living body with a property of extension at the time of an event. This extension can to some extent be described mathematically, starting from the Grassmann exterior product $\wedge$, allowing any $\mathrm{dx}$ to be extended as: $\mathrm{dx} \wedge \mathrm{dy} \wedge \mathrm{dz} \mathrm{dt}^{\wedge}$ [64].

The native emptiness which makes all natural living beings intimately feel incomplete would be what makes

\footnotetext{
${ }^{8}$ The energetist approach of Jung defined forms a complement with the causal mechanical approach. It considers that the biological energies tend to be organized for a goal, an exit, an end. This finality which is the why, the sense, is physically anticipated by advance waves. In contrast, the mechanical Fechner-Weber law referred to the bio-effect of a causal stimulus which arises according to delayed waves. Such a law loses its significance in energetist biology for example because of the sense of the physio-pathological pain which can be evaluated on an Analog Visual Scale, VAS.
} 
sensitive to and extensible in the structure of their 4D environment, at the time of events. Such a lack at the origin also pushes the being to seek a complementary self-similarity. This lack could be the basis of the relational energetics which applies to viruses just as they do to multicellular beings. Thus, certain signals present in the physical non-being can cause a supra-mechanical bio-resonance in simple beings. On this way, even if a virus is implanted in a mutated cell, there is always a native emptiness which will make this pathogen very sensitive to certain structural resonance frequencies.

\subsection{Resonance Frequencies for the Tumoral Cells of a Pancreatic Adenocarcinoma}

According to McInturff, 2007, [65], the “audio” range of frequencies to be applied in a Rife-Bare device to treat a "Cancer-adenocarcinoma", in addition to the sets of base frequencies for cancers in general, arranged by Tunney, [36], are the frequencies:

47, 2182, 2219, 832, 2084, 2127, 2160, 2452, 2876.

During the case study, Tunney’s sets of base frequencies were initially applied. The protocols brought into play a sweeping around the base frequencies. This sweeping of a few Hertz is generally organized like the striking of fly swatter, on the basis of a maximum frequency difference of innocent appearance, to finish head on, in order to alert the live target as late as possible, thus preventing it from escaping danger by changing its structure a little.

The base center frequencies used were: 10000, 11780, 21275, 17034, 11430, 10025, 6766, 6064, 5000, 3713, 3176, 3040, 2950, 2876, 2790, 2720, 2452, 2189, 2182, 2128, 2127, 2084, 2048, 2008, 1604, 1552, 1489, 880, 854, 800, 784, 776, 766, 728, 690, 683, 676, 666, 524, 464, 333, 120, 20. It can be seen that the $2128 \mathrm{~Hz}$ frequency suggested by Crane is in the list, but it is drowned in a mass and if it were alone could hardly act. A mechanical description of a single resonance in a reacting unicellular species would thus be of no use. A bio-resonance involving relational energetics could therefore be suitable for the living nature of the micro-organism, first occurring as a seizure, and able to proceed further until self-destruction.

The total negative form of a being's potentialities appears to be in the functional non-being. But it appears to be linked with the "useless" genome sequences, i.e. with the genetic mass inherited during evolution and which makes possible a regression toward previous forms. However, the cell's attitude which makes it organize its biological energies toward a aim depends on this form of a reality in negative, since it is determined by the sum \{being + non-being $\}$. The aim, or the finality, which forms the sense of the cell's action, would thus depend on environmental conditions associated with the functional non-being.

Non-mechanical resonance would thus cause a state of seizure in a pathogenic germ which may become lethal in the event of exact resonance, because it reverses the germ's finality. It causes a loss of sense which sends it backward in evolution. In the same way, a repeated negative event such as tar, asbestos fiber, repeated somatization of a negative emotion, i.e. accumulation of a negative self-similarity density in the 4D external medium, may lead a healthy cell to mutate, i.e. to become a unicellular species again, which, according to the theory of Davies, (2013), [66], is a backward step in the evolution.

Moreover, if the theory of Rife is accepted for convenience, a cell will have mutated because of a density disturbance in its functional non-being, and because of the presence of an oncogene virus in it. This virus has a resonance frequency which is related to its structured living matter and which may cause a destructive loss of sense: sent backward itself in its evolution, the germ undergoes a crisis of sense leading it to destruction. Once the virus, (i.e. the active structure within the tumoral cell), is destroyed, there is a discontinuity in the cellular antigens presentation. According to the theory of Pradeu, [6], this strongly supports the stimulation of the immune system so that the mutated cell is either repaired, or is destroyed. And when the self-similarity density of the applied signal becomes sufficient in the external medium, the signal may act, the tumor may duly disappear, and the endothelial cells of its blood network also disappear. This is also explained by the probable relation between the conservative forces of evolution in the autonomous nervous system and immune system, since both use the same molecules for information transfer and for control, as Smythies, (2011), [67], indicated it.

\section{Conclusion}

More than one year after its diagnosis of pancreatic cancer, and after more than six months of exposure to the radiofrequencies emitted by its PERL device, patient E.S. got several results. The first result was a fast recovery of its quality of life and functional health. The second one was a disappearance of its tumor in less than two 
months. The third one is a constant regression of the secondary infiltrations of the "mutated" cells on liver and pancreas. But the back increase of marker CA-19-9 level testifies to an activity recruit of the "mutated" cells, even if they are unable to gather and consequently incompetent to degrade the patient health. This last phenomenon is not opposed to a whole cure, according to a long-term process. Now with the only invasive treatment, the very frequent repetition of tumors leads finally to the death of the patients under generally painful conditions. Thus this case study showed that a bioelectromagnetic complementary treatment of this cancer could allow a victory.

A three-part explanation of the action mechanism of radiofrequency solitons upon pancreatic tumor is proposed: 1) According to physics, any non-linear system which emits a dissipative soliton is sensitive to the presence of an external structure of the same frequency. 2) According to biophysics, the exposure of the extracellular medium to radiofrequency solitons tends to produce a coherent structuring there. 3) According to empirical biology, the appearance of a soliton which can resonate with the structure of the micro-organism, tends to cause a seizure in it. This crisis corresponds to a disturbance of its living nature, with a loss of sense which makes the mutated unicellular cell unable to gather. And in case of purpose inversion, it results in self-destruction. Nevertheless, admitting that the resonance frequencies vary with the structural variations of the micro-organisms, considerable research remains to be carried out in order to determine the frequency ranges and sweeps to be applied to cover the majority of pancreatic adenocarcinoma varieties. Such frequency research appears to be a precondition before any clinical trial intended to prove the objective effectiveness of this therapy.

\section{References}

[1] Oettle, H. (2013) Recent Developments and Current Issues in the Treatment of Pancreatic Cancer. Journal of Cancer Therapy, 4, 13-27. http://dx.doi.org/10.4236/jct.2013.410A003

[2] Wolfgang, C.L., Herman, J.M., Lahreu, D.A.Z., Klein, A.P. and Erdek, M.A. (2013) Recent Progress in Pancreatic Cancer, CA. A Cancer Journal for Clinicians, 63, 318-348. http://dx.doi.org/10.1002/caac.21190

[3] Stathis, R.A. and Moore, M.J. (2010) Advanced Pancreatic Carcinoma: Current Treatment and Future Challenges. Nature Reviews, Clinical Oncology, 7, 163-172.

[4] Costa, J. (2012) Cancer, Encyclopaedia Britannica, Chicago.

[5] Cartlidge, E. (2013) Breast Cells in a Spin. Physics of Cancer, Physics World, 26, 28-32.

[6] Pradeu, Th. (2007) Immunology and Definition of Biological Identity. Doctorate Thesis, Paris1 Panthéon-Sorbonne University, Paris.

[7] Hammel, P. and André, Th. (2012) Pancreatic Cancer in Questions. Foundation ARCAD, Paris. www.fondationarcad.org

[8] Ball, P. (2013) Killing Me Softly. Physics of Cancer, Physics World, 26, 34-35.

[9] Tabor, E. (2002) Viruses and Liver Cancer, Perspectives in Medical Virology 6, Elsevier Sciences.

[10] Hassan, M.M., Li, D., El-Daab, A.S., Wolff, R.A., Bondy, M.L., Davila, M. and Abbruzzese, J.L. (2008) Association between Hepatitis B Virus ans Pancreatic Cancer. Journal of Clinical Oncology, 26, 4557-4562. http://dx.doi.org/10.1200/JCO.2008.17.3526

[11] Xu, B., Zheng, W.Y., Jin, D.Y., Wang, D.S., Liu, X.Y. and Qin, X.Y. (2012) Treatment of Pancreatic Cancer Using an Oncolytic Virus Harboring the Lipocalin 2-Gene. Cancer, 118, 5217-5226.

[12] Abbruzzese, J.L., Hruban, R.H., Yeo, C.J. and Campbell, E.R. (2007) Understanding Pancreatic Cancer, A Guide for Patient and Caregivers. The Lustgarten Foundation, New York. www.lustgarten.org

[13] Payne, D. and Kuster, N. (2013) Review and Evaluation of Beneficial Low-Level EMF Effects on Cancer Cells in with Respect to Potential Interaction Mechanisms. BioEM2013, Thessaloniki, 501-503.

[14] Pomonarev, O.A. and Fesenko, E.E. (2000) The Properties of Liquid Water in Electric and Magnetic Fields. Biofizika, 45, 389-398.

[15] Karimov, A.R. and Shcheglov, V.A. (2000) Role of Weak Energy Fluxes in the Formation of Coherent Structures in Macromolecular Media. Journal of Russian Laser Research, 21, 515-560.

[16] Fesenko, E.E., Popov, V.I., Novikov, V.V. and Khutsyan, S.S. (2002) Structure Formation in Water by the Action of Weak Magnetic Fields and Xenon, Electron Microscopy Analysis. Biophysika, 47, 398-394. (in Russian).

[17] Chen, G., Fenimore, P.W., Berendzen, J., Frauenfelder, H. and McMahon, B.H. (2009) The Role of Hydration Shells of Proteins. Los Alamos National Lab, Los Alamos. http://www.lanl.gov/orgs/adtsc/publications/science_highlights_2009/4chen.pdf 
[18] Jung, C.G. (1998) L’esprit et la vie. In: La réalité de l'âme, Structure et dynamique de l'inconscient [Mind and life. In: Reality of Soul, Structure and dynamics of the Unconscious], La Pochothèque, Le livre de poche, Librairie Générale Française, Paris, 366-385, Translated in French from: Seelen probleme der Gegenwart, 1931.

[19] Jimenez, H., Zimmerman, J., Brezovitch, I., Chen, D., Kuster, N., Capstick, M., Gong, Y., Barbault, A. and Pasche, B. (2013) In Vivo Tumor Growth Is Blocked by RF AM EMF and Is Associated with Peritumoral Fibrosis and Activation of the DAG/IP3 Pathway. BioEM2013, Thessaloniki, June 10-14, Abstract Collection, 08-1, 614-616.

[20] Lynes, B. (1993) The Cancer Cure That Worked. Marcus Books, San Francisco.

[21] Holland, A.G. (2010) Exposure of Human Pancreatic Cells to PEFT, 7 March. (Short Description in [22], 81-84). http://www.novobiotronics.com/

[22] Dubost, G. and Bellossi, A. (2008) Interprétation des effets in Vivo et in Vitro du rayonnement électromagnétique d'une antenne à plasma confiné [Interpretation of the in-Vivo and in-Vitro Effects of Exposure to a Confined Plasma Antenna Radiation]. Colloque Int. Sur la CEM et les journées scientifiques du CNFRS/URSI, 20-23 mai, Paris.

[23] Bellossi, A., Dubost, G., Bare, J. and Bardasano, J.L. (2010) Was Rife Right? A 32 Year Old Infection Cured in Two Hours by a Plasma Tube Irradiation. 6th International Workshop on Biological Effects of EM Fields, Bodrum, 10-14 October 2010, Abstract Book, 8-9.

[24] Bellossi, A. and Dubost, G. (2012) Thermal and Non Thermal Effects of Electromagnetic Fields on Biosystems. Lulu, Raleigh. http://www.lulu.com/

[25] Dubost, G., Bare, J., Holland, A. and Bellossi, F. (2013) Destructive Effects of Pulsed Electric Fields on Cancer Cells: The Microtubules Mechanical Resonance Clue. BioEM2013, Thessaloniki, June 10-14, Abstract Collection, PB224, 576-579.

[26] Scoon, A. (2001) The End of All Disease, Everyday Practical Electronics. April, Special Supplement, 1-13, Wimborne Publishing Ltd., Wimborne.

[27] Bare, J.E. (2005) Understanding Our Frequencies through Harmonic Associations. jbare@rifetechnologies.com

[28] Becker, R.O. and Marino, A.A. (1982) Electromagnetism and Life. State University of New York Press, Albany.

[29] http://www.rifeforum.com/

[30] Resonant Light, PERL. info@resonantlight.com

[31] de Santé, H.A. and HAS (2010) Guide ALD30, Cancer du pancreas [ALD30 Guide, Pancreatic Cancer]. (in French) http://www.has-sante.fr/

[32] Lévy, P. (2007) Adénocarcinome du pancréas: Le dosage du Ca 19-9 a-t-il un intérêt? [Pancreatic Adenocarcinoma: Is Test of Tumor Marker Ca 19-9 of Interest?] FMC-HGE, Post'U, Lyon. (in French)

[33] Prioré, A. (1963) Procédé et dispositif de production de rayonnements utilisables notamment pour le traitement des cellules vivantes [Procedure and Assemblage for Production of Radiation Especially Serviceable for the Treatment of Living Cells]. République Française, Brevet d'invention P.V. N 899 414, French Patent No. 1342772 , 7 October 1963.

[34] Prioré, A. (1968) Apparatus for Producing Radiations Penetrating Living Cells. US Patent No. 3368155.

[35] Perisse, E. (1984) Effets des ondes électromagnétiques et des champs magnétiques sur le cancer et la trypanosomiase expérimentale [Effects of EM Waves and Magnetic Fields on Cancer and Experimental Trypanosomiasis]. Doctoral Thesis, Université de Bordeaux, 2, N 83, 16 March 1984.

[36] Tunney, D. (2012) Protocols and Guidelines. Resonant Light Technology Inc., Courtenay. http://www.resonantlight.com/

[37] Bardos, C. (2012) Equations aux dérivées Partielles, Eq [Partial Derivative Equations]. Non-Linéaires, Encyclopédia Universalis, Paris. (in French)

[38] Kirson, E.D., Schneiderman, R.S., Dbaly, V., Tovarys, F., Vymazal, J., Izhaki, A., Mordechovich, D., Gurvich, Z., Shmueli, E., Goldsher, D., Wasseman, Y. and Palti, Y. (2009) Chemotherapeutic Treatment Efficacy and Sensitivity Are Increased by Adjuvant Alternating Electric Fields (TT Fields). BMC Medical Physics, 9, 1. http://www.biomedcentral.com/1756-6649/9/1

[39] Kiehn, R.M. (2012) Non-Equilibrium Systems and Irreversible Processes. Vol. 6, The Universal Effectiveness of Topological Thermodynamics, Lulu. http://www.lulu.com/kiehn

[40] Kiehn, R.M. (2013) The Finite Thermodynamic Topology of Photons. Proceedings of SPIE, 8832, V 883214, 1 October 2013. http://dx.doi.org/10.1117/12.2027316

[41] Akhmediev, N. (2004) Dissipative Solitons, Workshop on Mathematical Ideas in Nonlinear Optics: Guided Waves in Inhomogeneous Nonlinear Media. Edinburgh, July 19-23.

[42] Popp, F.A. and Klimek, W. (2007) Photon Sucking as an Essential Principle of Biological Regulation. In: Beloussov, 
L.V., Voeikov, V.L. and Martynyukeds, V.S., Eds., Biophotonics and Coherent Systems in Biology, Chapter 2, Springer, Berlin, 17-32

[43] Mesquita, M.V., Vasconcellos, A.R. and Luzzi, R. (2004) Considerations on Undistorted-Progressive X-Waves and Davydovsolitons, Fröhlich-Bose-Einstein Condensation, and Cherenkov-Like Effect in Biosystems. Brazilian Journal of Physics, 34, 489-503.

[44] Dubost, G., Bare, J.E. and Bellossi, F. (2014) Straight Astral Microtubule Mechanical Longitudinal Resonance Disrupt the Mitotic Spindle of Hepatocellular and Breast Adenocarcinoma cell in Vitro. Private Communication of a Paper to Be Published.

[45] Pasche, B. (2013) The Specificity of Modulation Frequencies in the Treatment of Cancer with Amplitude-Modulated Electromagnetic Fields. BioEM2013, Thessaloniki, June 10-14, Abstract Collection, p. 1-3, 4-5.

[46] Penrose, R. and Hameroff, S.R. (1995) What Gaps? Reply to Grush and Churchland. Journal of Consciousness Studies, 2, 99-112

[47] Hameroff, S. and Marcer, P. (1998) Quantum Computation in Brain Microtubules? The Penrose-Hameroff “Orch OR” Model of Consciousness (and Discussion). Philosophical Transactions: Mathematical, Physical and Engineering Sciences, 356, 1869-1896. Quantum Computation: Theory and Experiment (15 August 1998), Published by: The Royal Society. http://www.jstor.org/stable/55017

[48] Albrecht-Buehler, G. Cell Intelligence. Basic Science Administration. www.basic.northwestern.edu/g-buehler/summary.htm"

[49] Le Chapellier, P. and Matta, B. (2010) Cellular Perception and Active Penetration Depth for Pain Magnetotherapy. PIERS on Line, 6, 287-292.

[50] Pang, X.F. (2001) The Lifetime of the Soliton in the Improved Davydov Model at the Biological Temperature $300 \mathrm{~K}$ for Protein Molecules. The European Physical Journal B, 19, 297-316. http://dx.doi.org/10.1007/s100510170339

[51] Kiehn, R.M. (2008) The Photon as a Propagating Topological Singularity in the Lightcone. In: Non-Equilibrium Systems and Irreversible Processes, Adventures in Applied Topology, Vol. 4, Plasmas and Non-Equilibrium Electro- dynamics from a Perspective of Continuous Topological Evolution, Lulu. http://www.lulu.com/kiehn

[52] Le Chapellier, P. and Matta, B. (2011) Cellular Perception: When the Cell Model Includes a Sense Order Which Ensues from a Philosophy of Nature, the Signaling and Epigenetics Effects Which Can Result from Exposure to Magnetic Fields Are Described Better. Neurosciences and Medicine, 2, 161-177. http://www.scirp.org/journal/nm/ http://dx.doi.org/10.4236/nm.2011.23023

[53] Jung, C.G. (1998) Idem [18], p. 368.

[54] Jung, C.G. (1998) Idem [18], p. 375.

[55] Jung, C.G. (1998) La formation des symboles. In: L’énergétique psychique, La réalité de l'âme, Structure et dynamique de l'inconscient [Symbol Formation. In: Psychical Energetic, Reality of Soul, Structure and Dynamics of the Unconscious], 333, La Pochothèque, Le livre de poche, Librairie Générale Française, Paris, Translated in French from: Uber die Energetik der Seele, 1928.

[56] Jung, C.G. (1998) Pattern of behaviour et archétype, in:Réflexionsthéoriquessur la nature de l’inconscient. In: La réalité de l'âme, La réalité de l'âme, Structure et dynamique de l'inconscient [Archetype and Pattern of Behavior. In: Theoretical Reflectionsabout the Unconscious Nature, Reality of Soul, Structure and Dynamics of the Unconscious], 1035, La Pochothèque, Le livre de poche, LibrairieGénéraleFrançaise, Paris, 333, Translated in French from: Der Geist der Psychologie, 1946.

[57] Jung, C.G. (1998) Nominalisme et réalisme. In: Types et Symboles, La réalité de l'âme, Structure et dynamique de l'inconscient [Nominalism ans Realism. In: Types ans Symbols, Reality of Soul, Structure and Dynamics of the Unconscious], 252, La Pochothèque, Le livre de poche, Librairie Générale Française, Paris, Translated in French from: Psychological Types, 1921.

[58] Le Chapellier, P. and Matta, B. (2012) Cellular Psychology Assumption: Based upon Exterior Algebra, It Contributes to Explaining the Variability of the Bioeffects of Magnetic and Electromagnetic Fields. Neurosciences and Medicine, 3, 251-269. http://dx.doi.org/10.4236/nm.2012.33029 http://www.scirp.org/journal/nm

[59] Pang, X.F. (2006) The Conductivity Properties of Protons in Ice and Mechanism of Magnetization of Liquid Water, The European Physical Journal B, 49, 5-23. http://dx.doi.org/10.1140/epjb/e2006-00020-6

[60] Binhi, V.N. and Rubin, A.B. (2007) Magnetobiology: The kT Paradox and Possible Solutions. Electromagnetic Biology and Medicine, 26, 45-62. (Copyright: Informa Healthcare) http://dx.doi.org/10.1080/15368370701205677

[61] Le Chapellier, P. and Matta, B. (2013) Hypotheses on Cellular Perceptive Psychology. International Journal of Advance in Medical Sciences (AMS), 1-2, 29-35. http://www.seipub.org/ams/

[62] Whitehead, A.N. (1925) Science and the Modern World. The MacMillan Company, New York. 
[63] Merleau-Ponty, M. (1995) La Nature-Notes [Nature-Notes]. Cours au Collège de France, Seuil, Paris. (in French)

[64] Le Chapellier, P. and Matta, B. (2012) Cellular Psychology: Defined on an Exterior Algebra Using Complex Numbers, It Helps to Explain the Variablebioeffects. The Bioelectromagnetics Society, 34th Annual Meeting, Brisbane, 17-22 June 2012, Abstract Collection, 68-70.

[65] McInturff, B. (2007) The Consolidated Annotated Frequency List, CAFL. turf@mindspring.com

[66] Davies, P. (2013) Exposing Cancer's Deep Evolutionary Roots. Physics of Cancer, Physics World, 26, 37-40.

[67] Smythies, J. (2011) Some Aspects of the Normal Role of Neuromodulators in the Immune System. Neuroscience \& Medicine, 2, 275-281. http://www.scirp.org/journal/nm 